\title{
Examining Dense-Array Electroencephalographic Networks during Sleep - Partial or Non-Partial Correlations?
}

\author{
Calvin Kai-Ching $\mathrm{Yu}^{1 *}$, Wang-On $\mathrm{Li}^{1}$ \\ ${ }^{1}$ Department of Counselling and Psychology, Hong Kong Shue Yan University, Hong Kong
}

\begin{abstract}
Both partial and non-partial correlation methods have been utilized by researchers to construct dense-array electroencephalographic (dEEG) networks. Similarly, researchers have been using different protocols to preprocess data and minimize spurious correlations. This methodological study examined the extent to which the dEEG connectivity networks computed by the non-partial, first-order partial, and ( $\mathrm{N}-2)$-order $/ 254^{\text {th }}$-order partial correlation methods resemble each other, with two autoregressive integrative moving average (ARIMA) preprocessing models and three sample lengths being taken into consideration. Data were collected from two volunteers during the last 60 seconds of the second and fourth rapid-eyemovement epochs using a 256-channel electroencephalographic system and prior to correlation analyses, were preprocessed by either ARIMA $(40,1,1)$ or ARIMA $(20,1,1)$ transformation. The analyses demonstrate that the partial method, even at the first-order level, can substantially suppress the overall degree of connectivity in a network. Nevertheless, the moderate-tolarge rank-order correlation values comparing the similarities between the three network-construction methods casts doubt on the supposition that a network built upon partial correlations is fundamentally distinguished from that derived from nonpartial correlations.
\end{abstract}

Keywords: functional connectivity, high-density EEG, partial correlation, rapid eye movement, synchronous cortical activity

\section{INTRODUCTION}

Researchers have long been studying synchronous electroencephalographic (EEG) activities as neural correlates of conscious states. Their studies have yielded some findings, which have psychological implications. Pagel (2012), for instance, provided a comprehensive review of synchronous EEG manifestations of various conscious states associated with the sleep-wake cycle,

*Correspondence: calvinkcyuaymail.com

Calvin Kai-Ching Yu, Department of Counselling and Psychology, Hong Kong Shue Yan University, 10 Wai Tsui Crescent, Braemar Hill, North Point, Hong Kong

Received: 13 November 2017 Accepted: 5 December 2017

Sleep and Hypnosis

Journal homepage:

www.sleepandhypnosis.org

ISSN:1302-1192 (Print) 2458-9101 (Online) ranging from nonattentive waking states, through to hypnosis, psychedelic experience, and dreaming. He pointed out that lucid dreaming, as suggested by its enhanced alpha and gamma rhythms associated respectively with rapid-eye-movement (REM) sleep and focused meditation, is a state characteristic of both dreaming and wakefulness. Likewise, Dimitriadis, Laskaris, Del Rio-Portilla, and Koudounis (2009) noted an EEG segregation between the fronto-central and parietotemporal cortical regions during REM sleep, which probably reflects the functional disconnection between the waking cognition and perceptual systems.

With the advent of dense array electroencephalographic (dEEG) technology, the study of neural connectivity has been rendered far more sophisticated than a conventional connectivity study, which entails only a minimum of two intrahemispherically 
and two interhemispherically placed electrodes. A dEEG system today can gauge up to 256 channels covering almost the entire head surface, thus thoroughly visualizing the distribution of electrical potentials generated by different regions of the brain. This allows not only more proper plotting of synchronous networks during sleep but also more diverse methodologies in handling sleep EEG data. Thus far, only a few studies (e.g., Langheim, Murphy, Riedner, \&Tononi, 2011; Yu, 2015a) have attempted to construct dense sleep EEG networks using time-series statistics. By plotting significant partial correlations of all 32640 possible pairs of the 256 electrodes, Langheim et al. found a cluster of positive interactions in the right parieto-occipital region during REM sleep. Yu (2015a), on the other hand, used non-partial correlations, which resulted in positive connections over the anterio-frontocentral and occipito-temporo-cerebellar regions.

dEEG synchronous connectivity can be a new parameter or direction for investigating the variations in dreaming sleep. However, there are some methodological issues that should be resolved before more extensive $\mathrm{dEEG}$ connectivity sleep research can be conducted. This is peculiarly true when it is considered that $\mathrm{dEEG}$ sleep research is labor-intensive and computationally expensive in comparison with conventional polysomnography. In establishing dense EEG networks, researchers have been employing different protocols for data referencing, preprocessing, and analysis. This, at least in part, explains the differences in connectivity networks reported by different researchers. Langheim et al. (2011) referenced all data to the default electrode $\mathrm{Cz}$ placed at the vertex. Yu's (2014, 2015a, Yu \& Li, 2018) research group instead recommended applying a common average reference (the average of all 256 or 257 electrodes) in sleep research for more accurate localization of waveforms and reducing spurious correlations. It is worth noting that both the $\mathrm{Cz}$ and common average referencing approaches deviate from the American Academy of Sleep and Medicine's (AASM; Iber, Ancoli-Israel, Chesson, \& Quan, 2007) standard mastoid referencing.

Referencing to the mastoid electrode on either side as recommended by the AASM is inapt for network analysis in that it would create a systematic crescendo of electrical amplitude toward the opposite side of the head. Although this bias can be corrected by averaging the two mastoid electrodes of the sensor net (average mastoid reference), Yu and Li (2018) showed that the use of the average mastoid reference would cause the methodological artifact of the two mastoid electrodes being inversely and perfectly correlated. Additionally, the average mastoid reference tends to lower the strength of correlations for the electrodes over the mastoid process and temporal bone, wherein the inferior temporal cortex is located. Utilizing the mastoid electrodes as a reference would therefore lessen the sensitivity for detecting activities over the inferior temporal region, which is believed to play an important part in the neural network of dreaming sleep (Yu, 2001a, 2006).

Langheim et al. (2011) introduced the use of BoxJenkins autoregressive integrative moving average (ARIMA) modeling to remove the trend in each EEG time series before correlation analyses. The ARIMA modeling method can achieve stationary variance by suppressing autocorrelation structures and curtail spurious correlations resulting from the common trends or predictability of temporal structures. Langheim et al. proposed an optimal ARIMA model, which was composed of 40 AR orders $(A R=40)$, one differencing $\operatorname{order}(I=1)$, and one MA order $(M A=1)$. Yu $(2015 a)$ observed, however, that a sizable number of time series could not be fitted by the ARIMA $(40,1,1)$ model. Since the residuals for some channels could not be generated by the ARIMA $(40,1,1)$ modeling, the correlations for these unfitted channels could not be computed. As Yu and $\mathrm{Li}$ (2018) demonstrated, this rendered subsequent connectivity analyses unreliable in that some connections between certain critical regions for sleep, such as those between the parastriate region and the occipitotemporal junction (Yu, 2001a, 2006), could not be detected. They suggested, furthermore, that the $\operatorname{ARIMA}(20,1,1)$ model might constitute an alternative for sleep EEG data preprocessing because it effectively removed time structures to the extent very much like the ARIMA (40, 1, 1) model with virtually no unfittable channels for both early and late REM periods and different sample lengths.

Pearson correlation is the most classic measure of 
connectivity. Yu (2015a) applied non-partial Pearson correlations (zero-order correlation) in his study, whereas Langheim et al. (2011) computed a partial correlation coefficient for each pair of sensors with consideration of all the remaining 254 sensors ( $254^{\text {th }}$-order/(N-2)-order partial correlation). In their methodological studies, Jalili and Knyazeva (2011a, 2011b) introduced yet another approach and limited their computations to first-order partial correlations. Specifically, for each pair of sensors, they calculated ( $\mathrm{N}-2)$ correlations, with the remaining sensors being partialled out one at a time; the minimum among these $(\mathrm{N}-2)$ values was then chosen for further analyses.

As Jalili and Knyazeva (2011a, 2011b) averred, a correlation value may derive from direct interactions between two sensors and/or indirect connections via sequential pathways or common sources. In other words, it is assumed that non-partial correlation gives an aggregate estimate of both direct and indirect connections between two sensors, partial correlation measuring direct functional connectivity. This is, however, a theoretical supposition and the direct link or true value of the correlation between two sensors is a relative rather than absolute concept. At one end of the continuum is the correlation value with all possible indirect links being controlled, at the other end being the statistical procedure which partials out the single sensor that gives the minimum correlation value among all (N-2) first-order partial correlation values. Jalili and Knyazeva $(2011 \mathrm{a}, \mathrm{b})$ argued that synchronous networks built upon partial correlations fundamentally differ from those detected by non-partial correlations. This argument suggests that using different correlation methods to construct synchronous networks would give rise to distinct patterns of connections.

The study presented here aimed to examine the extent to which the first-order and ( $\mathrm{N}$-2)-order partial correlation methods would alter the rank-ordered pattern of sleep EEG connections computed based on non-partial correlations. If they could only suppress the overall strength of connectivity rather than alter the pattern of correlations, they might merely serve a similar function as do the preprocessing tactics for reducing spurious correlations. In addition, it should be noted that performing partial correlations for dEEC data with the standard sampling rate of $500 \mathrm{~Hz}$ can be very expensive in terms of computation time - approximately eight hours for 60-second data, for example. Reducing the length of each time series can substantially lower the cost of computation. Therefore, the effects of the partial and non-partial correlation methods were considered together with varied ARIMA models and lengths of time series. Specifically, it was hypothesized that correlation matrices generated using different partial/non-partial methods, ARIMA transformation models, and time lengths would share a similar pattern of relative connectivity magnitudes among sensors.

\section{METHOD}

Two volunteers' (Subject 1: male, 23 years old; Subject 2: female, 21 years old) sleep was monitored by a highdensity electroencephalographic system. The sensor net is composed of 256 index electrodes, covering almost the entire head, including areas on both cheeks and around both eyes. Data were sampled at $500 \mathrm{~Hz}$ and were referenced to the $\mathrm{Cz}$ electrode. Raw EEG data that were collected during the last 60 seconds of the second (REM2) and fourth (REM4) REM episodes of the second laboratory night and were relatively devoid of ocular and other artifacts were analyzed using the MATLAB and R statistical packages. The $\mathrm{Cz}$-referenced data were preprocessed by either ARIMA $(40,1,1)$ or ARIMA (20, $1,1)$ transformation before connectivity analyses. Pearson correlation coefficients of the 32640 pairs of electrodes ( $256 \times 255 / 2)$ were computed using the non-partial, firstorder partial, and $254^{\text {th }}$-order partial methods. After that, Spearman correlations were performed to assess the extent to which the rank orders of the Pearson correlation coefficients generated by the three methods resembled each other. Thorough comparisons of more ARIMA models in terms of their effects on subsequent connectivity analyses are available in Yu and Li's (2018) previous report. A detailed description of the current dEEG protocol can be found elsewhere (Yu, 2014, 2015a, 2015b, 2016a). The study was approved by the University Human Research Ethics Committee. 


\section{Analyses}

Table 1 lists the standard AASM derivations (see Column 1, F3 to O2) and nine other electrodes placed on the neuroanatomical regions especially relevant to sleep research (see Column 1, FT7 to TP10). For example, BA37 and BA40 are neural substrates for converting dream thoughts into dream images (see Yu, 2001a, 2001b, 2003, $2006,2007,2016 \mathrm{~b}$, for the neuroanatomical correlates of dreaming sleep). Tables 2-7 presents the Pearson correlation matrices of 60-s, REM4 data obtained with these selected channels and preprocessed by ARIMA (20, $1,1)$ modeling. In both Subject 1 (Tables $2 \& 3$ ) and Subject 2 (Tables $5 \& 6$ ), the first-order partial method significantly suppressed the correlations coefficients, z values ranging between 9.02 and 37.06, all $p$ values $<$
.001 . The effect of the $254^{\text {th }}$-order partial process (Tables $4 \& 7$ ), as compared with the first-order partial process (Tables $3 \& 6$ ), was even more robust, $z$ values ranging between 6.5 and 39.18, all $p$ values $<.001$. The magnitudes of all $254^{\text {th }}$-order partial correlations were minuscule. Nonetheless, the three correlation methods resulted in a similar pattern of relative magnitudes among the coefficients, the strongest connections being mostly clustered around P7, TP9, O1, P8, TP10, and O2.

The results for Spearman correlations are shown in Tables 8-11. The large rank-order correlation coefficients (> 0.5 ) indicated that varying the ARIMA parameters, the sampling rate, or even both did not substantially alter the rank-order sequence of coefficients between any two correlation matrices in both subjects and both REM stages,

Table 1. Electrode Positions and Corresponding Neuroanatomical Regions

\begin{tabular}{llll}
\hline $\mathbf{1 0 - 2 0}$ position & Electrode number & Brodmann area & Neuroanatomical area \\
\hline F3 & E36 & BA8 & Intermediate frontal \\
F4 & E224 & BA8 & Intermediate frontal \\
C3 & E59 & BA1-4 & Central \\
C4 & E183 & BA1-4 & Central \\
O1 & E116 & BA18 & Parastriate \\
O2 & E150 & BA18 & Parastriate \\
FT7 & E62 & BA47 & Orbitofrontal \\
FT8 & E211 & BA47 & Orbitofrontal \\
CP3 & E66 & BA40 & Supramarginal \\
CP4 & E164 & BA40 & Supramarginal \\
P7 & E96 & BA37 & Occipitotemporal \\
P8 & E170 & BA37 & Occipitotemporal \\
Cz & Reference & BA4 & Vertex \\
TP9 & E94 & BA20, BA21 & Mastoid/inferoposterior temporal \\
TP10 & E190 & BA20, BA21 & Mastoid/inferoposterior temporal \\
\hline
\end{tabular}

Table 2. Non-Partial Correlation Matrix for Cz Referenced, ARIMA $(20,1,1)$ Preprocessed, 60-s Data Extracted from Subject 1's REM4

\begin{tabular}{|c|c|c|c|c|c|c|c|c|c|c|c|c|c|}
\hline & F3 & F4 & C3 & C4 & 01 & 02 & FT7 & FT8 & CP3 & CP4 & P7 & P8 & TP9 \\
\hline F4 & 0.330 & & & & & & & & & & & & \\
\hline C3 & 0.320 & 0.162 & & & & & & & & & & & \\
\hline C4 & 0.193 & 0.313 & 0.128 & & & & & & & & & & \\
\hline 01 & 0.229 & 0.193 & 0.281 & 0.241 & & & & & & & & & \\
\hline 02 & 0.188 & 0.209 & 0.199 & 0.292 & 0.420 & & & & & & & & \\
\hline FT7 & 0.427 & 0.276 & 0.346 & 0.217 & 0.351 & 0.268 & & & & & & & \\
\hline FT8 & 0.289 & 0.380 & 0.195 & 0.354 & 0.290 & 0.312 & 0.335 & & & & & & \\
\hline CP3 & 0.270 & 0.147 & 0.394 & 0.143 & 0.312 & 0.234 & 0.335 & 0.186 & & & & & \\
\hline CP4 & 0.170 & 0.258 & 0.143 & 0.395 & 0.258 & 0.325 & 0.209 & 0.332 & 0.155 & & & & \\
\hline P7 & 0.284 & 0.196 & 0.348 & 0.225 & 0.469 & 0.357 & 0.401 & 0.278 & 0.377 & 0.229 & & & \\
\hline P8 & 0.204 & 0.243 & 0.188 & 0.338 & 0.363 & 0.422 & 0.272 & 0.361 & 0.204 & 0.351 & 0.318 & & \\
\hline TP9 & 0.315 & 0.235 & 0.317 & 0.245 & 0.467 & 0.367 & 0.446 & 0.367 & 0.330 & 0.240 & 0.483 & 0.346 & \\
\hline TP10 & 0.259 & 0.301 & 0.211 & 0.332 & 0.370 & 0.399 & 0.333 & 0.473 & 0.219 & 0.334 & 0.344 & 0.436 & 0.420 \\
\hline
\end{tabular}

Note. The 3 largest correlation coefficients are bolded. 
Table 3. First-Order Partial Correlation Matrix for Cz Referenced, ARIMA $(20,1,1)$ Preprocessed, 60-s Data Extracted from Subject 1's REM4

\begin{tabular}{|c|c|c|c|c|c|c|c|c|c|c|c|c|c|}
\hline & F3 & F4 & C3 & C4 & 01 & 02 & FT7 & FT8 & CP3 & CP4 & P7 & P8 & TP9 \\
\hline F4 & 0.176 & & & & & & & & & & & & \\
\hline C3 & 0.177 & 0.064 & & & & & & & & & & & \\
\hline C4 & 0.086 & 0.181 & 0.055 & & & & & & & & & & \\
\hline 01 & 0.090 & 0.077 & 0.140 & 0.113 & & & & & & & & & \\
\hline $\mathrm{O} 2$ & 0.078 & 0.090 & 0.083 & 0.157 & 0.228 & & & & & & & & \\
\hline FT7 & 0.239 & 0.121 & 0.203 & 0.094 & 0.169 & 0.120 & & & & & & & \\
\hline FT8 & 0.120 & 0.216 & 0.086 & 0.199 & 0.127 & 0.150 & 0.144 & & & & & & \\
\hline CP3 & 0.139 & 0.059 & 0.265 & 0.064 & 0.160 & 0.113 & 0.195 & 0.074 & & & & & \\
\hline CP4 & 0.072 & 0.133 & 0.067 & 0.280 & 0.125 & 0.188 & 0.098 & 0.186 & 0.075 & & & & \\
\hline P7 & 0.134 & 0.092 & 0.206 & 0.116 & 0.291 & 0.182 & 0.232 & 0.124 & 0.232 & 0.113 & & & \\
\hline P8 & 0.093 & 0.105 & 0.083 & 0.209 & 0.189 & 0.274 & 0.134 & 0.187 & 0.095 & 0.217 & 0.161 & & \\
\hline TP9 & 0.148 & 0.103 & 0.178 & 0.113 & 0.280 & 0.185 & 0.256 & 0.191 & 0.183 & 0.112 & 0.298 & 0.178 & \\
\hline TP10 & 0.120 & 0.150 & 0.091 & 0.185 & 0.192 & 0.233 & 0.163 & 0.276 & 0.095 & 0.190 & 0.179 & 0.262 & 0.233 \\
\hline
\end{tabular}

Note. The 3 largest correlation coefficients are bolded.

Table 4. $254^{\text {th }}$-Order Correlation Matrix for $C z$ Referenced, ARIMA $(20,1,1)$ Preprocessed, 60-s Data Extracted from Subject 1's REM4

\begin{tabular}{|c|c|c|c|c|c|c|c|c|c|c|c|c|c|}
\hline & F3 & F4 & C3 & $\mathrm{C} 4$ & 01 & $\mathrm{O} 2$ & FT7 & FT8 & CP3 & CP4 & P7 & P8 & TP9 \\
\hline F4 & 0.019 & & & & & & & & & & & & \\
\hline C3 & 0.004 & -0.008 & & & & & & & & & & & \\
\hline C4 & 0.003 & 0.017 & -0.007 & & & & & & & & & & \\
\hline 01 & -0.009 & 0.004 & -0.007 & -0.008 & & & & & & & & & \\
\hline 02 & 0.006 & 0.012 & -0.013 & -0.015 & 0.002 & & & & & & & & \\
\hline FT7 & 0.013 & 0.001 & 0.003 & -0.008 & 0.001 & -0.010 & & & & & & & \\
\hline FT8 & -0.001 & 0.017 & 0.013 & 0.006 & 0.001 & 0.002 & -0.003 & & & & & & \\
\hline CP3 & -0.004 & 0.006 & 0.049 & 0.000 & -0.008 & 0.003 & 0.009 & -0.008 & & & & & \\
\hline CP4 & 0.010 & -0.002 & 0.009 & 0.049 & -0.003 & 0.008 & 0.001 & 0.003 & 0.000 & & & & \\
\hline P7 & -0.002 & -0.005 & 0.001 & 0.005 & 0.012 & -0.006 & -0.001 & -0.001 & 0.016 & -0.008 & & & \\
\hline P8 & 0.004 & -0.009 & 0.002 & 0.013 & -0.003 & 0.032 & -0.008 & -0.008 & -0.004 & 0.012 & -0.008 & & \\
\hline TP9 & -0.008 & -0.014 & 0.007 & 0.007 & 0.016 & 0.002 & -0.007 & 0.017 & 0.008 & -0.004 & 0.032 & 0.001 & \\
\hline TP10 & -0.001 & -0.004 & 0.001 & 0.001 & -0.003 & 0.011 & -0.015 & 0.030 & -0.006 & 0.006 & 0.004 & 0.025 & 0.013 \\
\hline
\end{tabular}

Note. The 4 largest correlation coefficients are bolded.

Table 5. Non-Partial Correlation Matrix for Cz Referenced, ARIMA $(20,1,1)$ Preprocessed, 60-s Data Extracted from Subject 2's REM4

\begin{tabular}{|c|c|c|c|c|c|c|c|c|c|c|c|c|c|}
\hline & F3 & F4 & C3 & C4 & 01 & 02 & FT7 & FT8 & CP3 & CP4 & P7 & P8 & TP9 \\
\hline F4 & 0.377 & & & & & & & & & & & & \\
\hline C3 & 0.396 & 0.278 & & & & & & & & & & & \\
\hline C4 & 0.298 & 0.418 & 0.263 & & & & & & & & & & \\
\hline O1 & 0.288 & 0.253 & 0.327 & 0.306 & & & & & & & & & \\
\hline $\mathrm{O} 2$ & 0.274 & 0.296 & 0.271 & 0.360 & 0.482 & & & & & & & & \\
\hline FT7 & 0.430 & 0.324 & 0.378 & 0.291 & 0.365 & 0.327 & & & & & & & \\
\hline FT8 & 0.355 & 0.442 & 0.278 & 0.441 & 0.328 & 0.381 & 0.383 & & & & & & \\
\hline CP3 & 0.369 & 0.270 & 0.438 & 0.310 & 0.442 & 0.358 & 0.419 & 0.313 & & & & & \\
\hline CP4 & 0.269 & 0.359 & 0.260 & 0.437 & 0.328 & 0.381 & 0.283 & 0.413 & 0.308 & & & & \\
\hline P7 & 0.345 & 0.286 & 0.362 & 0.316 & 0.545 & 0.440 & 0.425 & 0.351 & 0.470 & 0.322 & & & \\
\hline P8 & 0.299 & 0.343 & 0.276 & 0.425 & 0.439 & 0.560 & 0.338 & 0.454 & 0.349 & 0.434 & 0.426 & & \\
\hline TP9 & 0.364 & 0.303 & 0.350 & 0.308 & 0.459 & 0.401 & 0.485 & 0.393 & 0.419 & 0.317 & 0.516 & 0.409 & \\
\hline TP10 & 0.316 & 0.346 & 0.279 & 0.398 & 0.407 & 0.483 & 0.383 & 0.507 & 0.351 & 0.396 & 0.412 & 0.557 & 0.462 \\
\hline
\end{tabular}

Note. The 3 largest correlation coefficients are bolded. 
Table 6. First-Order Partial Correlation Matrix for Cz Referenced, ARIMA $(20,1,1)$ Preprocessed, 60-s Data Extracted from Subject 2's REM4

\begin{tabular}{|c|c|c|c|c|c|c|c|c|c|c|c|c|c|}
\hline & F3 & $\mathrm{F} 4$ & $\mathrm{C} 3$ & $\mathrm{C4}$ & 01 & $\mathrm{O} 2$ & FT7 & FT8 & $\mathrm{CP3}$ & CP4 & P7 & P8 & TP9 \\
\hline F4 & 0.201 & & & & & & & & & & & & \\
\hline C3 & 0.227 & 0.154 & & & & & & & & & & & \\
\hline C4 & 0.151 & 0.237 & 0.137 & & & & & & & & & & \\
\hline 01 & 0.117 & 0.111 & 0.159 & 0.143 & & & & & & & & & \\
\hline $\mathrm{O} 2$ & 0.120 & 0.124 & 0.128 & 0.163 & 0.272 & & & & & & & & \\
\hline FT7 & 0.238 & 0.155 & 0.205 & 0.144 & 0.169 & 0.157 & & & & & & & \\
\hline FT8 & 0.160 & 0.236 & 0.136 & 0.243 & 0.141 & 0.174 & 0.184 & & & & & & \\
\hline CP3 & 0.197 & 0.141 & 0.249 & 0.178 & 0.250 & 0.188 & 0.223 & 0.153 & & & & & \\
\hline CP4 & 0.133 & 0.193 & 0.143 & 0.276 & 0.160 & 0.184 & 0.145 & 0.226 & 0.173 & & & & \\
\hline P7 & 0.161 & 0.136 & 0.179 & 0.153 & 0.306 & 0.222 & 0.217 & 0.168 & 0.260 & 0.159 & & & \\
\hline P8 & 0.139 & 0.148 & 0.137 & 0.219 & 0.215 & 0.321 & 0.168 & 0.231 & 0.176 & 0.224 & 0.200 & & \\
\hline TP9 & 0.168 & 0.133 & 0.160 & 0.139 & 0.230 & 0.195 & 0.236 & 0.177 & 0.197 & 0.150 & 0.262 & 0.198 & \\
\hline TP10 & 0.150 & 0.159 & 0.123 & 0.202 & 0.175 & 0.260 & 0.181 & 0.267 & 0.166 & 0.196 & 0.195 & 0.331 & 0.219 \\
\hline
\end{tabular}

Note. The 3 largest correlation coefficients are bolded.

Table 7. $254^{\text {th }}$-Order Correlation Matrix for Cz Referenced, ARIMA $(20,1,1)$ Preprocessed, 60-s Data Extracted from Subject 2's REM4

\begin{tabular}{|c|c|c|c|c|c|c|c|c|c|c|c|c|c|}
\hline & F3 & F4 & C3 & $\mathrm{C} 4$ & 01 & 02 & FT7 & FT8 & CP3 & CP4 & P7 & P8 & TP9 \\
\hline F4 & 0.001 & & & & & & & & & & & & \\
\hline C3 & 0.008 & 0.003 & & & & & & & & & & & \\
\hline C4 & -0.003 & 0.013 & -0.006 & & & & & & & & & & \\
\hline O1 & -0.008 & -0.011 & -0.004 & -0.011 & & & & & & & & & \\
\hline 02 & 0.005 & 0.012 & -0.004 & -0.011 & 0.018 & & & & & & & & \\
\hline FT7 & -0.001 & -0.004 & 0.002 & -0.012 & -0.005 & 0.008 & & & & & & & \\
\hline FT8 & -0.003 & -0.006 & 0.002 & 0.002 & -0.003 & -0.009 & 0.001 & & & & & & \\
\hline CP3 & 0.003 & -0.010 & 0.027 & 0.014 & 0.014 & -0.006 & 0.008 & -0.004 & & & & & \\
\hline CP4 & 0.002 & 0.009 & 0.004 & 0.036 & 0.001 & -0.010 & 0.002 & 0.010 & 0.004 & & & & \\
\hline P7 & 0.003 & 0.001 & -0.008 & 0.001 & 0.036 & -0.008 & 0.002 & 0.003 & 0.014 & -0.005 & & & \\
\hline P8 & 0.003 & 0.001 & 0.008 & 0.017 & 0.002 & 0.046 & -0.007 & -0.008 & -0.005 & 0.012 & 0.011 & & \\
\hline TP9 & -0.009 & -0.010 & -0.003 & -0.004 & 0.008 & 0.005 & 0.004 & -0.000 & -0.011 & 0.010 & 0.009 & 0.009 & \\
\hline TP10 & -0.002 & -0.007 & -0.007 & 0.000 & -0.002 & 0.005 & 0.002 & 0.013 & 0.009 & -0.004 & -0.007 & 0.024 & 0.014 \\
\hline
\end{tabular}

Note. The 3 largest correlation coefficients are bolded.

Table 8. Spearman Correlation Coefficients Comparing the 256-channel Correlation Matrices between Non-Partial and Partial Correlations (Subject 1's REM2)

\begin{tabular}{|c|c|c|c|c|c|c|c|c|c|c|c|c|c|c|c|c|c|}
\hline & $\begin{array}{l}\mathrm{Np} \\
(20,1,1) \\
15 s\end{array}$ & $\begin{array}{l}1^{\text {st }} \text { order } \\
(20,1,1) \\
15 s\end{array}$ & $\begin{array}{l}254^{\text {th }} \\
\text { order } \\
(20,1,1) \\
15 s\end{array}$ & $\begin{array}{l}\mathrm{Np} \\
(40,1,1) \\
15 s\end{array}$ & $\begin{array}{l}1^{\text {st }} \text { order } \\
(40,1,1) \\
15 s\end{array}$ & $\begin{array}{l}254^{\text {th }} \\
\text { order } \\
(40,1,1) \\
15 s\end{array}$ & $\begin{array}{l}\mathrm{Np} \\
(20,1,1) \\
30 \mathrm{~s}\end{array}$ & $\begin{array}{l}1^{\text {st }} \text { order } \\
(20,1,1) \\
30 \text { s }\end{array}$ & $\begin{array}{l}254^{\text {th }} \\
\text { order } \\
(20,1,1) \\
30 \text { s }\end{array}$ & $\begin{array}{l}\mathrm{Np} \\
(40,1,1) \\
30 \mathrm{~s}\end{array}$ & $\begin{array}{l}1^{\text {st }} \text { order } \\
(40,1,1) \\
30 \mathrm{~s}\end{array}$ & $\begin{array}{l}254^{\text {th }} \\
\text { order } \\
(40,1,1) \\
30 \text { s }\end{array}$ & $\begin{array}{l}\mathrm{Np} \\
(20,1,1) \\
60 \mathrm{~s}\end{array}$ & $\begin{array}{l}1^{\text {st }} \text { order } \\
(20,1,1) \\
60 \mathrm{~s}\end{array}$ & $\begin{array}{l}254^{\text {th }} \\
\text { order } \\
(20,1,1) \\
60 \mathrm{~s}\end{array}$ & $\begin{array}{l}\mathrm{Np} \\
(40,1,1) \\
60 \mathrm{~s}\end{array}$ & $\begin{array}{l}1^{\text {st }} \text { order } \\
(40,1,1) \\
60 \mathrm{~s}\end{array}$ \\
\hline $1^{\text {st }}$ order $(20,1,1) 15 \mathrm{~s}$ & 0.928 & & & & & & & & & & & & & & & & \\
\hline $254^{\text {th }}$ order $(20,1,1) 15 \mathrm{~s}$ & 0.309 & 0.411 & & & & & & & & & & & & & & & \\
\hline $\mathrm{Np}(40,1,1) 15 \mathrm{~s}$ & 0.998 & 0.931 & 0.318 & & & & & & & & & & & & & & \\
\hline $1^{\text {st }} \operatorname{order}(40,1,1) 15 \mathrm{~s}$ & 0.915 & 0.997 & 0.417 & 0.924 & & & & & & & & & & & & & \\
\hline $254^{\text {th }}$ order $(40,1,1) 15 \mathrm{~s}$ & 0.316 & 0.420 & 0.990 & 0.322 & 0.425 & & & & & & & & & & & & \\
\hline $\mathrm{Np}(20,1,1) 30 \mathrm{~s}$ & 0.992 & 0.903 & 0.283 & 0.987 & 0.885 & 0.289 & & & & & & & & & & & \\
\hline $1^{\text {st }}$ order $(20,1,1) 30 \mathrm{~s}$ & 0.950 & 0.977 & 0.359 & 0.949 & 0.968 & 0.367 & 0.947 & & & & & & & & & & \\
\hline $254^{\text {th }}$ order $(20,1,1) 30 \mathrm{~s}$ & 0.225 & 0.273 & 0.250 & 0.244 & 0.295 & 0.300 & 0.220 & 0.265 & & & & & & & & & \\
\hline $\mathrm{Np}(40,1,1) 30 \mathrm{~s}$ & 0.994 & 0.912 & 0.293 & 0.993 & 0.898 & 0.301 & 0.998 & 0.950 & 0.247 & & & & & & & & \\
\hline $1^{\text {st }}$ order $(40,1,1) 30 \mathrm{~s}$ & 0.943 & 0.983 & 0.366 & 0.947 & 0.979 & 0.376 & 0.933 & 0.996 & 0.295 & 0.943 & & & & & & & \\
\hline $254^{\text {th }}$ order $(40,1,1) 30 \mathrm{~s}$ & 0.241 & 0.288 & 0.309 & 0.260 & 0.308 & 0.353 & 0.242 & 0.292 & 0.826 & 0.246 & 0.294 & & & & & & \\
\hline $\mathrm{Np}(20,1,1) 60 \mathrm{~s}$ & 0.987 & 0.888 & 0.265 & 0.981 & 0.869 & 0.271 & 0.997 & 0.935 & 0.212 & 0.993 & 0.919 & 0.235 & & & & & \\
\hline $1^{\text {st }}$ order $(20,1,1) 60 \mathrm{~s}$ & 0.948 & 0.966 & 0.331 & 0.946 & 0.955 & 0.338 & 0.948 & 0.992 & 0.254 & 0.950 & 0.987 & 0.282 & 0.944 & & & & \\
\hline $254^{\text {th }}$ order $(20,1,1) 60 \mathrm{~s}$ & 0.164 & 0.197 & 0.045 & 0.185 & 0.221 & 0.085 & 0.160 & 0.189 & 0.382 & 0.191 & 0.223 & 0.379 & 0.156 & 0.186 & & & \\
\hline $\mathrm{Np}(40,1,1) 60 \mathrm{~s}$ & 0.989 & 0.894 & 0.272 & 0.986 & 0.879 & 0.279 & 0.995 & 0.936 & 0.227 & 0.996 & 0.926 & 0.241 & 0.998 & 0.945 & 0.174 & & \\
\hline $1^{\text {st }}$ order $(40,1,1) 60 \mathrm{~s}$ & 0.941 & 0.971 & 0.337 & 0.944 & 0.965 & 0.345 & 0.935 & 0.989 & 0.271 & 0.943 & 0.991 & 0.289 & 0.930 & 0.996 & 0.205 & 0.939 & \\
\hline $254^{\text {th }}$ order $(40,1,1) 60 \mathrm{~s}$ & 0.357 & 0.442 & 0.621 & 0.368 & 0.448 & 0.626 & 0.359 & 0.446 & 0.322 & 0.370 & 0.453 & 0.385 & 0.356 & 0.448 & 0.038 & 0.362 & 0.453 \\
\hline
\end{tabular}

Note. $\mathrm{Np}=$ non-partial correlation matrix, $1^{\text {st }}$ order $=$ first-order partial correlation matrix, $254^{\text {th }}$ order $=\mathrm{N}-2$ partial correlation matrix, $(40,1,1)=$ ARIMA $(40,1,1)$ preprocessed correlation matrix

$(20,1,1)=$ ARIMA $(20,1,1)$ preprocessed correlation matrix, $15 \mathrm{~s}=$ calculated based on 15 -second data, $30 \mathrm{~s}=$ calculated based on 30 -second data, $60 \mathrm{~s}=$ calculated based on 60 -second data 
especially those comparing non-partial and first-order partial correlation matrices. The rank-order correlation coefficient comparing any non-partial and first-order partial correlation matrices was very large, with the overall average value of 0.938 . For example, the rank-order correlation coefficient between the non-partial correlation matrix for the $15-\mathrm{s}$, ARIMA $(20,1,1)$ processed data and the firstorder partial correlation matrix for the 60-s, ARIMA (40, 1, 1) processed data was 0.941 in Subject 1's REM2 (see Table
8) and 0.926 in Subject2's REM4 (see Table 11). By contrast, $254^{\text {th }}$-order partial correlation matrices considerably differed from their non-partial and first-order partial counterparts. Nevertheless, most of the rank-order correlation coefficients exhibited a moderate effect size (> 0.3). The overall average rank-order correlation coefficient comparing non-partial and $254^{\text {th }}$-order partial correlation matrices was 0.316 , that comparing first-order and $254^{\text {th }}$ order partial correlation matrices being 0.391 .

Table 9. Spearman Correlation Coefficients Comparing the 256-channel Correlation Matrices between Non-Partial and Partial Correlations (Subject 1's REM4)

\begin{tabular}{|c|c|c|c|c|c|c|c|c|c|c|c|c|c|c|c|c|c|}
\hline & $\begin{array}{l}\mathrm{Np} \\
(20,1,1) \\
15 s\end{array}$ & $\begin{array}{l}1^{\text {st }} \text { order } \\
(20,1,1) \\
15 \mathrm{~s}\end{array}$ & $\begin{array}{l}254^{\text {th }} \\
\text { order } \\
(20,1,1) \\
15 s\end{array}$ & $\begin{array}{l}\mathrm{Np} \\
(40,1,1) \\
15 \mathrm{~s}\end{array}$ & $\begin{array}{l}1^{\text {st }} \text { order } \\
(40,1,1) \\
15 s\end{array}$ & $\begin{array}{l}254^{\text {th }} \\
\text { order } \\
(40,1,1) \\
15 s\end{array}$ & $\begin{array}{l}\mathrm{Np} \\
(20,1,1) \\
30 \mathrm{~s}\end{array}$ & $\begin{array}{l}1^{\text {st }} \text { order } \\
(20,1,1) \\
30 \mathrm{~s}\end{array}$ & $\begin{array}{l}254^{\text {th }} \\
\text { order } \\
(20,1,1) \\
30 \mathrm{~s}\end{array}$ & $\begin{array}{l}\mathrm{Np} \\
(40,1,1) \\
30 \mathrm{~s}\end{array}$ & $\begin{array}{l}1^{\text {st }} \text { order } \\
(40,1,1) \\
30 \mathrm{~s}\end{array}$ & $\begin{array}{l}254^{\text {th }} \\
\text { order } \\
(40,1,1) \\
30 \mathrm{~s}\end{array}$ & $\begin{array}{l}\mathrm{Np} \\
(20,1,1) \\
60 \mathrm{~s}\end{array}$ & $\begin{array}{l}1^{\text {st }} \text { order } \\
(20,1,1) \\
60 \mathrm{~s}\end{array}$ & $\begin{array}{l}254^{\text {th }} \\
\text { order } \\
(20,1,1) \\
60 \mathrm{~s}\end{array}$ & $\begin{array}{l}\mathrm{Np} \\
(40,1,1) \\
60 \mathrm{~s}\end{array}$ & $\begin{array}{l}1^{\text {st }} \text { order } \\
(40,1,1) \\
60 \mathrm{~s}\end{array}$ \\
\hline $1^{\text {st }}$ order $(20,1,1) 15 \mathrm{~s}$ & 0.952 & & & & & & & & & & & & & & & & \\
\hline $254^{\text {th }}$ order $(20,1,1) 15 \mathrm{~s}$ & 0.344 & 0.414 & & & & & & & & & & & & & & & \\
\hline $\mathrm{Np}(40,1,1) 15 \mathrm{~s}$ & 0.998 & 0.951 & 0.360 & & & & & & & & & & & & & & \\
\hline $1^{\text {st }} \operatorname{order}(40,1,1) 15 \mathrm{~s}$ & 0.954 & 0.954 & 0.457 & 0.961 & & & & & & & & & & & & & \\
\hline $254^{\text {th }}$ order $(40,1,1) 15 \mathrm{~s}$ & 0.331 & 0.431 & 0.961 & 0.338 & 0.424 & & & & & & & & & & & & \\
\hline $\mathrm{Np}(20,1,1) 30 \mathrm{~s}$ & 0.993 & 0.959 & 0.311 & 0.990 & 0.937 & 0.311 & & & & & & & & & & & \\
\hline $1^{\text {st }}$ order $(20,1,1) 30 \mathrm{~s}$ & 0.951 & 0.991 & 0.368 & 0.950 & 0.948 & 0.384 & 0.966 & & & & & & & & & & \\
\hline $254^{\text {th }}$ order $(20,1,1) 30 \mathrm{~s}$ & 0.373 & 0.431 & 0.784 & 0.391 & 0.482 & 0.744 & 0.365 & 0.436 & & & & & & & & & \\
\hline $\mathrm{Np}(40,1,1) 30 \mathrm{~s}$ & 0.991 & 0.960 & 0.324 & 0.991 & 0.942 & 0.325 & 0.998 & 0.968 & 0.381 & & & & & & & & \\
\hline $1^{\text {st }}$ order $(40,1,1) 30 \mathrm{~s}$ & 0.957 & 0.973 & 0.397 & 0.963 & 0.982 & 0.389 & 0.960 & 0.984 & 0.471 & 0.967 & & & & & & & \\
\hline $254^{\text {th }}$ order $(40,1,1) 30 \mathrm{~s}$ & 0.358 & 0.450 & 0.746 & 0.376 & 0.450 & 0.771 & 0.366 & 0.455 & 0.957 & 0.374 & 0.457 & & & & & & \\
\hline $\mathrm{Np}(20,1,1) 60 \mathrm{~s}$ & 0.977 & 0.950 & 0.283 & 0.969 & 0.909 & 0.291 & 0.987 & 0.954 & 0.329 & 0.982 & 0.936 & 0.340 & & & & & \\
\hline $1^{\text {st }} \operatorname{order}(20,1,1) 60 \mathrm{~s}$ & 0.928 & 0.964 & 0.338 & 0.923 & 0.917 & 0.354 & 0.941 & 0.970 & 0.397 & 0.939 & 0.950 & 0.415 & 0.956 & & & & \\
\hline $254^{\text {th }}$ order $(20,1,1) 60 \mathrm{~s}$ & 0.382 & 0.435 & 0.645 & 0.399 & 0.485 & 0.606 & 0.374 & 0.440 & 0.808 & 0.389 & 0.473 & 0.767 & 0.356 & 0.443 & & & \\
\hline $\mathrm{Np}(40,1,1) 60 \mathrm{~s}$ & 0.974 & 0.952 & 0.299 & 0.970 & 0.914 & 0.304 & 0.986 & 0.955 & 0.347 & 0.984 & 0.942 & 0.355 & 0.999 & 0.956 & 0.375 & & \\
\hline $1^{\text {st }} \operatorname{order}(40,1,1) 60 \mathrm{~s}$ & 0.928 & 0.956 & 0.359 & 0.928 & 0.934 & 0.360 & 0.937 & 0.964 & 0.421 & 0.939 & 0.961 & 0.425 & 0.947 & 0.995 & 0.468 & 0.953 & \\
\hline $254^{\text {th }}$ order $(40,1,1) 60 \mathrm{~s}$ & 0.368 & 0.456 & 0.604 & 0.377 & 0.447 & 0.627 & 0.376 & 0.460 & 0.761 & 0.388 & 0.463 & 0.795 & 0.368 & 0.466 & 0.954 & 0.375 & 0.468 \\
\hline
\end{tabular}

Note. $\mathrm{Np}=$ non-partial correlation matrix, $1^{\text {st }}$ order $=$ first-order partial correlation matrix, $254^{\text {th }}$ order $=\mathrm{N}-2$ partial correlation matrix, $(40,1,1)=A R I M A(40,1,1)$ preprocessed correlation matrix,

$(20,1,1)=$ ARIMA $(20,1,1)$ preprocessed correlation matrix, $15 \mathrm{~s}=$ calculated based on 15 -second data, $30 \mathrm{~s}=$ calculated based on 30 -second data, 60s $=$ calculated based on 60 -second data.

Table 10. Spearman Correlation Coefficients Comparing the 256-channel Correlation Matrices between Non-Partial and Partial Correlations (Subject 2's REM2)

\begin{tabular}{|c|c|c|c|c|c|c|c|c|c|c|c|c|c|c|c|c|c|}
\hline & $\begin{array}{l}\mathrm{Np} \\
(20,1,1) \\
15 \mathrm{~s}\end{array}$ & $\begin{array}{l}1^{\text {st }} \text { order } \\
(20,1,1) \\
15 s\end{array}$ & $\begin{array}{l}254^{\text {th }} \\
\text { order } \\
(20,1,1) \\
15 \mathrm{~s}\end{array}$ & $\begin{array}{l}\mathrm{Np} \\
(40,1,1) \\
15 s\end{array}$ & $\begin{array}{l}1^{\text {st }} \text { order } \\
(40,1,1) \\
15 \mathrm{~s}\end{array}$ & $\begin{array}{l}254^{\text {th }} \\
\text { order } \\
(40,1,1) \\
15 s\end{array}$ & $\begin{array}{l}\mathrm{Np} \\
(20,1,1) \\
30 \mathrm{~s}\end{array}$ & $\begin{array}{l}1^{\text {st }} \text { order } \\
(20,1,1) \\
30 \text { s }\end{array}$ & $\begin{array}{l}254^{\text {th }} \\
\text { order } \\
(20,1,1) \\
30 \mathrm{~s}\end{array}$ & $\begin{array}{l}\mathrm{Np} \\
(40,1,1) \\
30 \mathrm{~s}\end{array}$ & $\begin{array}{l}1^{\text {st }} \text { order } \\
(40,1,1) \\
30 \mathrm{~s}\end{array}$ & $\begin{array}{l}254^{\text {th }} \\
\text { order } \\
(40,1,1) \\
30 \mathrm{~s}\end{array}$ & $\begin{array}{l}\mathrm{Np} \\
(20,1,1) \\
60 \mathrm{~s}\end{array}$ & $\begin{array}{l}1^{\text {st }} \text { order } \\
(20,1,1) \\
60 \mathrm{~s}\end{array}$ & $\begin{array}{l}254^{\text {th }} \\
\text { order } \\
(20,1,1) \\
60 \mathrm{~s}\end{array}$ & $\begin{array}{l}\mathrm{Np} \\
(40,1,1) \\
60 \mathrm{~s}\end{array}$ & $\begin{array}{l}1^{\text {st }} \text { order } \\
(40,1,1) \\
60 \text { s }\end{array}$ \\
\hline $1^{\text {st }} \operatorname{order}(20,1,1) 15 \mathrm{~s}$ & 0.960 & & & & & & & & & & & & & & & & \\
\hline $254^{\text {th }}$ order $(20,1,1) 15 \mathrm{~s}$ & 0.303 & 0.405 & & & & & & & & & & & & & & & \\
\hline $\mathrm{Np}(40,1,1) 15 \mathrm{~s}$ & 0.998 & 0.966 & 0.317 & & & & & & & & & & & & & & \\
\hline $1^{\text {st }}$ order $(40,1,1) 15 \mathrm{~s}$ & 0.955 & 0.997 & 0.411 & 0.961 & & & & & & & & & & & & & \\
\hline $254^{\text {th }}$ order $(40,1,1) 15 \mathrm{~s}$ & 0.296 & 0.383 & 0.958 & 0.303 & 0.391 & & & & & & & & & & & & \\
\hline $\mathrm{Np}(20,1,1) 30 \mathrm{~s}$ & 0.990 & 0.947 & 0.272 & 0.989 & 0.941 & 0.276 & & & & & & & & & & & \\
\hline $1^{\text {st }} \operatorname{order}(20,1,1) 30 \mathrm{~s}$ & 0.939 & 0.973 & 0.345 & 0.945 & 0.972 & 0.340 & 0.951 & & & & & & & & & & \\
\hline $254^{\text {th }}$ order $(20,1,1) 30 \mathrm{~s}$ & 0.326 & 0.420 & 0.777 & 0.341 & 0.427 & 0.742 & 0.316 & 0.407 & & & & & & & & & \\
\hline $\mathrm{Np}(40,1,1) 30 \mathrm{~s}$ & 0.987 & 0.948 & 0.285 & 0.988 & 0.946 & 0.289 & 0.999 & 0.955 & 0.330 & & & & & & & & \\
\hline $1^{\text {st }} \operatorname{order}(40,1,1) 30 \mathrm{~s}$ & 0.931 & 0.968 & 0.353 & 0.940 & 0.971 & 0.349 & 0.944 & 0.998 & 0.415 & 0.951 & & & & & & & \\
\hline $254^{\text {th }}$ order $(40,1,1) 30 \mathrm{~s}$ & 0.319 & 0.399 & 0.741 & 0.335 & 0.409 & 0.769 & 0.322 & 0.406 & 0.956 & 0.328 & 0.411 & & & & & & \\
\hline $\mathrm{Np}(20,1,1) 60 \mathrm{~s}$ & 0.989 & 0.937 & 0.260 & 0.987 & 0.930 & 0.267 & 0.997 & 0.938 & 0.301 & 0.995 & 0.929 & 0.310 & & & & & \\
\hline $1^{\text {st }} \operatorname{order}(20,1,1) 60 \mathrm{~s}$ & 0.958 & 0.973 & 0.322 & 0.961 & 0.969 & 0.324 & 0.968 & 0.989 & 0.376 & 0.971 & 0.984 & 0.381 & 0.964 & & & & \\
\hline $254^{\text {th }}$ order $(20,1,1) 60 \mathrm{~s}$ & 0.350 & 0.441 & 0.652 & 0.367 & 0.449 & 0.620 & 0.342 & 0.430 & 0.818 & 0.357 & 0.437 & 0.779 & 0.340 & 0.429 & & & \\
\hline $\mathrm{Np}(40,1,1) 60 \mathrm{~s}$ & 0.987 & 0.939 & 0.269 & 0.988 & 0.935 & 0.278 & 0.997 & 0.942 & 0.311 & 0.997 & 0.937 & 0.320 & 0.998 & 0.967 & 0.352 & & \\
\hline $1^{\text {st }} \operatorname{order}(40,1,1) 60 \mathrm{~s}$ & 0.950 & 0.969 & 0.327 & 0.958 & 0.970 & 0.331 & 0.962 & 0.989 & 0.382 & 0.969 & 0.989 & 0.388 & 0.956 & 0.997 & 0.436 & 0.963 & \\
\hline $254^{\text {th }}$ order $(40,1,1) 60 \mathrm{~s}$ & 0.339 & 0.416 & 0.620 & 0.357 & 0.427 & 0.642 & 0.343 & 0.424 & 0.780 & 0.358 & 0.432 & 0.808 & 0.345 & 0.429 & 0.961 & 0.352 & 0.435 \\
\hline
\end{tabular}

Note. $\mathrm{Np}=$ non-partial correlation matrix, $1^{\text {st }}$ order $=$ first-order partial correlation matrix, $254^{\text {th }}$ order $=\mathrm{N}-2$ partial correlation matrix, $(40,1,1)=\mathrm{ARIMA}(40,1,1)$ preprocessed correlation matrix,

$(20,1,1)=$ ARIMA $(20,1,1)$ preprocessed correlation matrix, $15 \mathrm{~s}=$ calculated based on 15 -second data, $30 \mathrm{~s}=$ calculated based on 30 -second data, $60 \mathrm{~s}=$ calculated based on 60 -second data. 
Table 11. Spearman Correlation Coefficients Comparing the 256-channel Correlation Matrices between Non-Partial and Partial Correlations (Subject 2's REM4)

\begin{tabular}{|c|c|c|c|c|c|c|c|c|c|c|c|c|c|c|c|c|c|}
\hline & $\begin{array}{l}\mathrm{Np} \\
(20,1,1) \\
15 \mathrm{~s}\end{array}$ & $\begin{array}{l}1^{\text {st }} \text { order } \\
(20,1,1) \\
15 s\end{array}$ & $\begin{array}{l}254^{\text {th }} \\
\text { order } \\
(20,1,1) \\
15 s\end{array}$ & $\begin{array}{l}\mathrm{Np} \\
(40,1,1) \\
15 \mathrm{~s}\end{array}$ & $\begin{array}{l}1^{\text {st }} \text { order } \\
(40,1,1) \\
15 \mathrm{~s}\end{array}$ & $\begin{array}{l}254^{\text {th }} \\
\text { order } \\
(40,1,1) \\
15 s\end{array}$ & $\begin{array}{l}\mathrm{Np} \\
(20,1,1) \\
30 \mathrm{~s}\end{array}$ & $\begin{array}{l}1^{\text {st }} \text { order } \\
(20,1,1) \\
30 \text { s }\end{array}$ & $\begin{array}{l}254^{\text {th }} \\
\text { order } \\
(20,1,1) \\
30 \mathrm{~s}\end{array}$ & $\begin{array}{l}\mathrm{Np} \\
(40,1,1) \\
30 \mathrm{~s}\end{array}$ & $\begin{array}{l}1^{\text {st }} \text { order } \\
(40,1,1) \\
30 \mathrm{~s}\end{array}$ & $\begin{array}{l}254^{\text {th }} \\
\text { order } \\
(40,1,1) \\
30 \mathrm{~s}\end{array}$ & $\begin{array}{l}\mathrm{Np} \\
(20,1,1) \\
60 \mathrm{~s}\end{array}$ & $\begin{array}{l}1^{\text {st }} \text { order } \\
(20,1,1) \\
60 \mathrm{~s}\end{array}$ & $\begin{array}{l}254^{\text {th }} \\
\text { order } \\
(20,1,1) \\
60 \mathrm{~s}\end{array}$ & $\begin{array}{l}\mathrm{Np} \\
(40,1,1) \\
60 \mathrm{~s}\end{array}$ & $\begin{array}{l}1^{\text {st }} \text { order } \\
(40,1,1) \\
60 \mathrm{~s}\end{array}$ \\
\hline $1^{\text {st }} \operatorname{order}(20,1,1) 15 \mathrm{~s}$ & 0.927 & & & & & & & & & & & & & & & & \\
\hline $254^{\text {th }}$ order $(20,1,1) 15 \mathrm{~s}$ & 0.318 & 0.400 & & & & & & & & & & & & & & & \\
\hline $\mathrm{Np}(40,1,1) 15 \mathrm{~s}$ & 0.998 & 0.928 & 0.323 & & & & & & & & & & & & & & \\
\hline $1^{\text {st }} \operatorname{order}(40,1,1) 15 \mathrm{~s}$ & 0.928 & 0.962 & 0.428 & 0.932 & & & & & & & & & & & & & \\
\hline $254^{\text {th }}$ order $(40,1,1) 15 \mathrm{~s}$ & 0.304 & 0.408 & 0.961 & 0.308 & 0.402 & & & & & & & & & & & & \\
\hline $\mathrm{Np}(20,1,1) 30 \mathrm{~s}$ & 0.992 & 0.915 & 0.284 & 0.989 & 0.899 & 0.280 & & & & & & & & & & & \\
\hline $1^{\text {st }} \operatorname{order}(20,1,1) 30 \mathrm{~s}$ & 0.941 & 0.980 & 0.349 & 0.939 & 0.942 & 0.356 & 0.946 & & & & & & & & & & \\
\hline $254^{\text {th }}$ order $(20,1,1) 30 \mathrm{~s}$ & 0.346 & 0.413 & 0.777 & 0.351 & 0.446 & 0.735 & 0.335 & 0.416 & & & & & & & & & \\
\hline $\mathrm{Np}(40,1,1) 30 \mathrm{~s}$ & 0.991 & 0.916 & 0.290 & 0.991 & 0.902 & 0.286 & 0.999 & 0.946 & 0.341 & & & & & & & & \\
\hline $1^{\text {st }}$ order $(40,1,1) 30 \mathrm{~s}$ & 0.949 & 0.967 & 0.371 & 0.951 & 0.973 & 0.357 & 0.943 & 0.985 & 0.442 & 0.946 & & & & & & & \\
\hline $254^{\text {th }}$ order $(40,1,1) 30 \mathrm{~s}$ & 0.328 & 0.421 & 0.742 & 0.334 & 0.415 & 0.765 & 0.328 & 0.424 & 0.959 & 0.334 & 0.425 & & & & & & \\
\hline $\mathrm{Np}(20,1,1) 60 \mathrm{~s}$ & 0.973 & 0.911 & 0.268 & 0.968 & 0.884 & 0.269 & 0.983 & 0.942 & 0.314 & 0.980 & 0.932 & 0.314 & & & & & \\
\hline $1^{\text {st }}$ order $(20,1,1) 60 \mathrm{~s}$ & 0.905 & 0.965 & 0.324 & 0.902 & 0.928 & 0.330 & 0.907 & 0.979 & 0.382 & 0.905 & 0.963 & 0.389 & 0.936 & & & & \\
\hline $254^{\text {th }}$ order $(20,1,1) 60 \mathrm{~s}$ & 0.365 & 0.427 & 0.647 & 0.370 & 0.459 & 0.608 & 0.354 & 0.430 & 0.814 & 0.360 & 0.455 & 0.773 & 0.351 & 0.436 & & & \\
\hline $\mathrm{Np}(40,1,1) 60 \mathrm{~s}$ & 0.986 & 0.924 & 0.274 & 0.986 & 0.900 & 0.275 & 0.995 & 0.952 & 0.321 & 0.996 & 0.946 & 0.321 & 0.999 & 0.934 & 0.360 & & \\
\hline $1^{\text {st }} \operatorname{order}(40,1,1) 60 \mathrm{~s}$ & 0.926 & 0.974 & 0.339 & 0.927 & 0.961 & 0.335 & 0.921 & 0.984 & 0.399 & 0.923 & 0.986 & 0.394 & 0.931 & 0.995 & 0.454 & 0.935 & \\
\hline $254^{\text {th }}$ order $(40,1,1) 60 \mathrm{~s}$ & 0.348 & 0.436 & 0.616 & 0.353 & 0.430 & 0.633 & 0.349 & 0.439 & 0.779 & 0.356 & 0.441 & 0.805 & 0.356 & 0.448 & 0.961 & 0.357 & 0.448 \\
\hline
\end{tabular}

Note. $\mathrm{Np}=$ non-partial correlation matrix, $1^{\text {st }}$ order $=$ first-order partial correlation matrix, $254^{\text {th }}$ order $=\mathrm{N}-2$ partial correlation matrix, $(40,1,1)=\mathrm{ARIMA}(40,1,1)$ preprocessed correlation matrix,

$(20,1,1)=$ ARIMA $(20,1,1)$ preprocessed correlation matrix, $15 \mathrm{~s}=$ calculated based on 15 -second data, $30 \mathrm{~s}=$ calculated based on 30 -second data, $60 \mathrm{~s}=$ calculated based on 60 -second data.

\section{DISCUSSION}

The study presented here compared the dEEG connectivity matrices generated by the non-partial, firstorder partial, and (N-2)-order partial correlation methods, taking two ARIMA preprocessing models and three sample lengths into consideration. The results indicate that a partial correlation process for dEEG analysis, even at the first-order level, can substantially suppress the degree of connectivity. The effect of the $(\mathrm{N}-2)$-order partial process in the dEEG context is even more dramatic in that the correlation coefficients can be infinitesimal after partialling out the remaining 254 sensors. Notwithstanding the significant suppression effect, the first-order partial process does not greatly alter the pattern of the relative magnitudes among the 32640 possible connections. The first-order partial method, which involves identifying the minimum value from 254 correlation values for each of the 32640 possible connections in each dataset, necessitates computer programing. Indeed, as shown by the present analyses, the rank orderings of the non-partial and firstorder partial correlation matrices are so similar that researchers may need to consider whether writing such an algorithm is cost-effective.
By contrast, the (N-2)-order partial process would yield a correlation matrix that is only moderately similar to its corresponding first-order partial and non-partial correlation matrices. This suggests that it might detect a network akin to, but not the same as, those discerned by the first-order partial and non-partial methods. Partialling out $\mathrm{N}-2$ sensors in $\mathrm{dEEG}$ could result in improbably small coefficients; that is, a close-to-zero coefficient is unreasonable for a pair of neighboring or functionally related sensors. Accordingly, certain adjustments are necessary in further analyses, such as lowering the threshold when plotting dEEG networks. Future studies may explore the optimal number of sensors being partialled out that could produce more realistic coefficients for dEEG connectivity research.

Changing the ARIMA parameters, the sampling rate, or even both do not appear to significantly alter a dEEC network. On the one hand, researchers may, during the initial stage, search for a dEEG network using shorter time series to reduce computation time. On the other hand, however, choosing appropriate ARIMA parameters that can fit all 256 time-series data is still essential for examining synchronous dEEG activities. It should be noted that in the study presented here, the correlation coefficients were computed with the unfitted channels being 
excluded. As mentioned previously, ARIMA $(40,1,1)$ preprocessing may create a considerable number of unfitted channels, which may in turn lead to an underestimation of connectivity in certain neuroanatomical regions (Yu, 2015a; Yu \& Li, 2018).

Non-partial correlations, theoretically, account for aggregate connectivity contributed by both direct and indirect links, partial correlations being assumed to signify direct coupling. The study presented here demonstrates, nevertheless, that even the partial

\section{References}

Dimitriadis, S. I, Laskaris, N. A., Del Rio-Portilla, Y., \& Koudounis, G. Ch. (2009). Characterizing dynamic functional connectivity across sleep stages from EEG. Brain Topography, 22, 119-133.

Iber, C., Ancoli-Israel, S., Chesson, A., \& Quan, S. F. (2007). The AASM manual for the scoring of sleep and associated events: Rules, terminology and technical specifications. Winchester, IL: American Academy of Sleep Medicine.

Jalili, M., \& Knyazeva, M. G. (2011a). Constructing brain functional networks from EEG: Partial and unpartial correlations. Journal of Integrative Neuroscience, 10, 213-232.

Jalili, M., \& Knyazeva, M. G. (2011b). EEG-based functional networks in schizophrenia. Computers in Biology and Medicine, 41, 1178-1186.

Langheim, F. J. P., Murphy, M., Riedner, B. A., \& Tononi, G. (2011). Functional connectivity in slow-wave sleep: Identification of synchronous cortical activity during wakefulness and sleep using time series analysis of electroencephalographic data. Journal of Sleep Research, 20, 496-505.

Pagel, J. F. (2012). The synchronous electrophysiology of conscious states. Dreaming, 22, 173-191.

Yu, C. K.-C. (2001a). Neuroanatomical correlates of dreaming: The supramarginal gyrus controversy (dream work). NeuroPsychoanalysis, 3, 47-59.

Yu, C. K.-C. (2001b). Neuroanatomical correlates of dreaming, II: The ventromesial frontal region controversy (dream instigation). Neuro-Psychoanalysis, 3, 193-201.

Yu, C. K.-C. (2003). Neuroanatomical correlates of dreaming, III: The frontal-lobe controversy (dream censorship). NeuroPsychoanalysis, 5, 159-169. correlation procedure that controls the effects of all remaining 254 electrodes would produce a connectivity pattern that can be, to a moderate extent, matched with a network without eliminating ancillary links. Accordingly, whether a network built upon partial correlations is fundamentally different from that constructed via non-partial correlations is debatable. For large-scale dEEG studies, perhaps, non-partial correlation is probably a more feasible and effective approach.

Yu, C. K.-C. (2006). Memory loss is not equal to loss of dream experience: A clinicoanatomical study of dreaming in patients with posterior brain lesions. Neuro-Psychoanalysis, 8, 191-198.

Yu, C. K.-C. (2007). Cessation of dreaming and ventromesial frontal-region infarcts. Neuro-Psychoanalysis, 9, 85-92.

Yu, C. K.-C. (2014). Toward $100 \%$ dream retrieval by rapid-eyemovement sleep awakening: A high-density electroencephalographic study. Dreaming, 24, 1-17.

Yu, C. K.-C. (2015a). A high-density electroencephalographic study of synchronous networks during rapid-eye-movement sleep. Dreaming, 25, 1-17.

Yu, C. K.-C. (2015b). The vicissitudes of affective valence across the night: A high-density electroencephalographic study. Dreaming, 25, 274-290.

Yu, C. K.-C. (2016a). We dream typical themes every single night. Dreaming, 26, 319-329.

$\mathrm{Yu}$, C. K.-C. (2016b). A neuroanatomical framework for understanding dream content. Sleep and Hypnosis, 18, 82-91.

Yu, C. K.-C., \& Li, W.-O. (2018). A fundamental question about the application of high-density electroencephalography and timeseries analysis in examining synchronous networks during sleep - Does the use of different referencing and data preprocessing methods really matter? Sleep and Hypnosis, 20, 67-84. 